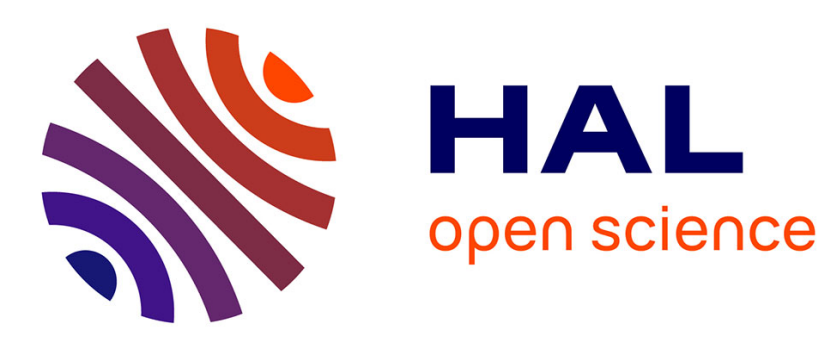

\title{
Variation of the conformal radius
}

Steffen Rhode, Michel Zinsmeister

\section{To cite this version:}

Steffen Rhode, Michel Zinsmeister. Variation of the conformal radius. Journal d'analyse mathématique, 2004, Vol 92, pp.105-115. hal-00079993

\section{HAL Id: hal-00079993 \\ https://hal.science/hal-00079993}

Submitted on 14 Jun 2006

HAL is a multi-disciplinary open access archive for the deposit and dissemination of scientific research documents, whether they are published or not. The documents may come from teaching and research institutions in France or abroad, or from public or private research centers.
L'archive ouverte pluridisciplinaire HAL, est destinée au dépôt et à la diffusion de documents scientifiques de niveau recherche, publiés ou non, émanant des établissements d'enseignement et de recherche français ou étrangers, des laboratoires publics ou privés. 


\title{
Variation of the conformal radius
}

\author{
Steffen Rohde*and Michel Zinsmeister
}

Preliminary version, July 28, 2001

\begin{abstract}
We study the change of the conformal radius $r(U)$ of a simply connected planar domain $U$ versus the subdomain $U_{\epsilon}$ consisting of the points of distance at least $\epsilon$ to $\partial U$. We show that the smallest exponent $\lambda$ such that $r(U)-r\left(U_{\epsilon}\right)=O\left(\epsilon^{\lambda}\right)$ satisfies $0.59<\lambda<0.91$. We also show that a well-known conjecture implies $\lambda=2(\sqrt{2}-1)$.
\end{abstract}

\section{Statement of the results}

Let $U$ be a simply connected proper subdomain of the plane and $z_{0} \in U$. By the Riemann mapping theorem there exists a unique holomorphic bijection $\varphi$ from the unit disk $\mathbb{D}$ onto $U$ such that $\varphi(0)=z_{0}, \varphi^{\prime}(0)>0$ and we will call this map the Riemann map of $\left(U, z_{0}\right)$. The positive real number $\varphi^{\prime}(0)$ is called the conformal radius $r\left(U, z_{0}\right)$, since $r\left(U, z_{0}\right)$ can also be characterized as the real number $r>0$ such that there exists $\psi: U \rightarrow D(0, r)$ with $\psi\left(z_{0}\right)=0, \psi^{\prime}\left(z_{0}\right)=1$. For $0<\epsilon<d\left(z_{0}, \partial U\right)$ (the distance from $z_{0}$ to $\partial U$ ) we define $U_{\epsilon}$ as the connected component of $z_{0}$ of the set $\{z \in U ; d(z, \partial U)>\epsilon\}$. It is again a simply connected domain containing $z_{0}$ : the main goal of this paper is the study of the speed of convergence of $r\left(U_{\epsilon}, z_{0}\right)$ to $r\left(U, z_{0}\right)$ as $\epsilon \rightarrow 0$. Our first result is a simple consequence of the Koebe distortion theorem:

Proposition 1. For $0<\epsilon<d\left(z_{0}, \partial U\right)$ we have

$$
0<r\left(U, z_{0}\right)-r\left(U_{\epsilon}, z_{0}\right) \leq 4 \sqrt{r\left(U, z_{0}\right)} \sqrt{\epsilon}
$$

Proof: By the Koebe theorem (see [4], p.9),

$$
\frac{1}{4}\left(1-|z|^{2}\right)\left|\varphi^{\prime}(z)\right| \leq d(\varphi(z), \partial U) \leq\left(1-|z|^{2}\right)\left|\varphi^{\prime}(z)\right|
$$

and

$$
\left|\varphi^{\prime}(z)\right| \geq \frac{1-|z|}{(1+|z|)^{3}} \varphi^{\prime}(0)
$$

for all $z \in \mathbb{D}$. From these two inequalities we deduce

*Partially supported by NSF Grant DMS-9970398. 


$$
d(\varphi(z), \partial U) \geq \frac{1}{16}(1-|z|)^{2} \varphi^{\prime}(0) .
$$

Let $D_{\epsilon}=\varphi^{-1}\left(U_{\epsilon}\right)$ : inequality (1.4) implies that $D_{\epsilon} \supset D(0, r)$ if $\frac{1}{16}(1-r)^{2} \varphi^{\prime}(0) \geq$ $\epsilon$. We fix such a $r$ and let $\varphi_{\epsilon}$ be the Riemann map of $\left(U_{\epsilon}, z_{0}\right)$; we have $\varphi_{\epsilon}=\varphi \circ h_{\epsilon}$ where $h_{\epsilon}$ is the Riemann map of $D_{\epsilon}$. Since $\mathbb{D} \supset D_{\epsilon} \supset D(0, r)$, Schwarz' lemma implies that $1>h_{\epsilon}^{\prime}(z) \geq r$. The left inequality in (1.1) follows, as well as the inequality $r\left(U, z_{0}\right)-r\left(U_{\epsilon}, z_{0}\right) \leq(1-r) r\left(U, z_{0}\right)$. The right inequality in (1.1) follows by optimizing in $r$.

In the sequel we will say that $U$ is normalized if $0 \in U, r(U, 0)=1$. We will also denote as usual by $S$ the set of injective holomorphic maps $\varphi: \mathbb{D} \rightarrow \mathbb{C}$ such that $\varphi(0)=0, \varphi^{\prime}(0)=1$.

If $U$ is smooth, it is easy to see that $\sqrt{\epsilon}$ in (1.1) can be replaced by $C \epsilon$, where $C$ depends on the smoothness of $U$ only. The objective of this paper is to show that the "trivial bound" $\sqrt{\epsilon}$ in (1.1) can be improved for every simply connected domain $U$, but that it cannot be improved up to $\epsilon$. More precisely, we will prove the following

Theorem 1. For all normalized simply connected domains $U$ there exists $\epsilon_{0}>0$ such that

$$
r(U, 0)-r\left(U_{\epsilon}, 0\right) \leq \epsilon^{0.59} \quad \text { for all } \epsilon<\epsilon_{0} .
$$

Conversely, there exists a normalized domain $U$ and $\epsilon_{0}>0$ such that

$$
r(U, 0)-r\left(U_{\epsilon}, 0\right) \geq \epsilon^{0.91} \quad \text { for all } \epsilon<\epsilon_{0} .
$$

Furthermore we will establish, assuming the validity of a well-known conjecture about integral means (Kraetzer's conjecture, see section 4) the correct critical exponent. More precisely we will show that, assuming Kraetzer's conjecture is true and putting $\lambda=2(\sqrt{2}-1)=0.828 .$. , then (1.5) is true with 0.59 replaced by $\lambda-\alpha$ for any $\alpha>0$, while for any $\delta>0$ there exists a normalized $U$ such that (1.6) is true with 0.91 replaced by $\lambda+\alpha$.

In section 2 we reduce the problem to an area estimate. In section 3 we use lacunary series to construct a domain satisfying (1.6). In section 4 we show that the problem is equivalent to a certain estimate for integral means of derivatives of conformal maps, and use this connection to prove (1.5) and to determine the critical exponent (assuming Kraetzer's conjecture).

The authors wish to thank Adrien Douady for adressing the problem of the speed of convergence of the conformal radius and the second author wishes to thank Martine Babillot for an instructive conversation concerning (1.6). 


\section{Reduction of the problem}

We consider a normalized domain $U$; we recall that $D_{\epsilon}=\varphi^{-1}\left(U_{\epsilon}\right)$. If $\Omega$ is a Borel subset of the plane we will denote by $|\Omega|$ its area (2-dimensional Lebesgue measure).

Proposition 2. There exists a universal constant $C>1$ such that

$$
\frac{1}{C}\left|\mathbb{D} \backslash D_{\epsilon}\right| \leq r(U, 0)-r\left(U_{\epsilon}, 0\right) \leq C\left|\mathbb{D} \backslash D_{\epsilon}\right|
$$

\section{Proof:}

a) Left inequality: We recall that $\varphi_{\epsilon}=\varphi \circ h_{\epsilon}$ where $\varphi_{\epsilon}, h_{\epsilon}$ are the Riemann maps of $\left(U_{\epsilon}, 0\right),\left(D_{\epsilon}, 0\right)$ respectively. Since $\varphi^{\prime}(0)=1$ we have $r(U, 0)-$ $r\left(U_{\epsilon}, 0\right)=1-h_{\epsilon}^{\prime}(0)=1-a_{1}(\epsilon)$ if we write $h_{\epsilon}(z)=\sum_{n \geq 1} a_{n}(\epsilon) z^{n}$. Using $\left|D_{\epsilon}\right|=\pi \sum_{n \geq 1} n\left|a_{n}(\epsilon)\right|^{2}$, we obtain $2 \pi\left(1-a_{1}(\epsilon)\right) \geq \pi\left(1-a_{1}(\epsilon)^{2}\right)=|\mathbb{D}|-$ $\left|D_{\epsilon}\right|+\pi \sum_{n \geq 2} n\left|a_{n}(\epsilon)\right|^{2} \geq\left|\mathbb{D} \backslash D_{\epsilon}\right|$, and the left inequality in (2.1) is proven with $C=2 \pi$.

b) Right inequality: We consider the usual dyadic decomposition of the disk: for each dyadic interval $I$ of length $2 \pi 2^{-n}$ on the unit circle we consider the corresponding "square" $Q=\left\{z \in \mathbb{D} ; z /|z| \in I, 1-|z| \leq 2^{-n}\right\}$, and we denote $T(Q)$ the "top half" of $Q$ so that the $T(Q)^{\prime} s$ form (modulo their boundaries) a partition of the disk. Notice also that each dyadic square $Q$ is the union of $T(Q)$ and two dyadic squares (the "children" of $Q$ ). For each $Q$ we also denote by $z_{Q}$ the middle of the top edge of $Q$. We then consider the usual random walk along the $z_{Q}^{\prime} s$, choosing at each stage $z_{Q}$ one of the two children of $Q$. We stop the walk as soon as $\left(1-\left|z_{Q}\right|^{2}\right)\left|\varphi^{\prime}\left(z_{Q}\right)\right| \leq \epsilon^{\prime}$ for some $\epsilon^{\prime}$ which will be chosen in term of $\epsilon$ later. This provides us with a family of disjoint squares $\left(Q_{j}\right)$; fix such a square $Q_{j}$ and let $z \in T(Q)$ where $Q$ is the "father" of $Q_{j}$. By Koebe theorem and the maximality of $Q_{j}$ there exists a universal $C>0$ such that $d(\varphi(z), \partial U) \geq C\left(1-\left|z_{Q}\right|^{2}\right)\left|\varphi^{\prime}\left(z_{Q}\right)\right| \geq C \epsilon^{\prime}=\epsilon$ if $\epsilon^{\prime}=\epsilon / C$. It follows that $\mathbb{D} \backslash D_{\epsilon} \subset \cup_{j} Q_{j}$ and, writing $D_{\epsilon}^{\prime}=\mathbb{D} \backslash \cup_{j} Q_{j}$, we have $r(\mathbb{D}, 0)-r\left(D_{\epsilon}, 0\right) \leq$ $r(\mathbb{D}, 0)-r\left(D_{\epsilon}^{\prime}, 0\right)$. The proof will thus be complete if we can prove:

$$
\begin{gathered}
r(\mathbb{D}, 0)-r\left(D_{\epsilon}^{\prime}, 0\right) \leq C\left|\mathbb{D} \backslash D_{\epsilon}^{\prime}\right|, \\
\left|\mathbb{D} \backslash D_{\epsilon}^{\prime}\right| \leq C\left|\mathbb{D} \backslash D_{\epsilon}\right| .
\end{gathered}
$$

We begin with (2.3). It suffices to show that for each $j$ there exists a square $Q \subset Q_{j}$ such that $|Q| \geq c\left|Q_{j}\right|$ and $Q \subset \mathbb{D} \backslash D_{\epsilon}$. To prove this we first use the fact [4] that there exists a geodesic $\gamma$ (for the hyperbolic metric) joining $z_{Q_{j}}$ to $Q_{j} \cap \partial \mathbb{D}$ and $C>0$ such that the euclidean length of $\gamma$ is $\leq C\left(1-\left|z_{Q_{j}}\right|\right)$. We then consider the first point $z$ on the geodesic such that $d(\varphi(z), \partial U)=\frac{\epsilon}{2}$. Between $z_{Q_{j}}$ and $z$ we thus have $C \epsilon \geq d(\varphi(\zeta), \partial U) \geq \frac{\epsilon}{2}$, from which it follows 
that the hyperbolic distance between $z_{Q_{j}}$ and $z$ is less than a (universal) $C>0$. It thus suffices to choose a square centered at $z$ with side $\epsilon^{\prime}\left(1-\left|z_{Q}\right|\right), \epsilon^{\prime}$ small enough (independently of $j$ ).

Next we prove (2.2): we consider the increasing sequence of domains $\Omega_{n}$ with $\Omega_{0}=D_{\epsilon}^{\prime}$ and $\Omega_{n+1}=\Omega_{n} \cup Q_{j_{n}}$ where $Q_{j_{n}}$ is (one of) the largest square(s) in $\mathbb{D} \backslash \Omega_{n}$. Let $m=\omega\left(0, \partial Q_{j_{n}}, \Omega_{n}\right)$, the harmonic measure of $\partial Q_{j_{n}}$ from 0 in $\Omega_{n}$. Let $\left.r_{n} \in\right] 0,1$ [ be such that the top edge of $Q_{j_{n}}$ lies in the circle of center 0 and radius $r_{n}$. We have $\Omega_{n} \supset D\left(0, r_{n}\right)$ so, by the maximum principle,

$$
\begin{aligned}
C \operatorname{diam}\left(Q_{j_{n}}\right) & \geq \omega\left(0, \partial Q_{j_{n}}, \mathbb{D} \backslash Q_{j_{n}}\right) \geq m \\
& \geq \omega\left(0, \partial Q_{j_{n}}, D\left(0, r_{n}\right)\right) \geq c \operatorname{diam}\left(Q_{j_{n}}\right)
\end{aligned}
$$

Let $\varphi_{n}$ be the Riemann map of $\left(\Omega_{n}, 0\right)$. By construction, the $\Omega_{n}^{\prime} s$ are uniform quasidisks, so that the $\varphi_{n}$ have uniformly quasiconformal extensions to $\mathbb{C}$. Therefore $Q_{n}^{\prime}=\varphi_{n+1}^{-1}\left(Q_{j_{n}}\right)$ is a $K$ - quasidisk for some $K$ not depending on $n$, and there are points $\zeta_{n} \in \partial \mathbb{D}$ and $\left.\rho_{n} \in\right] 0,1\left[\right.$ with $D\left(\zeta_{n}, c \rho_{n}\right) \subset Q_{n}^{\prime} \subset D\left(\zeta_{n}, C \rho_{n}\right)$. The maximum principle and (2.4) shows that $m \sim \rho$ and hence $\left|Q_{n}^{\prime}\right| \sim\left|Q_{j_{n}}\right|$. Since $1-r(\mathbb{D} \backslash D(1, \rho), 0) \sim \rho^{2}$, it follows that $1-\left(\varphi_{j_{n+1}}^{-1} \circ \varphi_{j_{n}}\right)^{\prime}(0) \sim\left|Q_{n}^{\prime}\right| \sim\left|Q_{j_{n}}\right|$ and $\left(\varphi_{n+1}^{-1} \circ \varphi_{n}\right)^{\prime}(0)=\frac{\varphi_{n}^{\prime}(0)}{\varphi_{n+1}^{\prime}(0)}$, which implies that

$$
\varphi_{n+1}^{\prime}(0)-\varphi_{n}^{\prime}(0)=\varphi_{n+1}^{\prime}(0)\left(1-\frac{\varphi_{n}^{\prime}(0)}{\varphi_{n+1}^{\prime}(0)}\right) \sim 1-\frac{\varphi_{n}^{\prime}(0)}{\varphi_{n+1}^{\prime}(0)} \sim|Q| .
$$

Finally $1-\varphi_{0}^{\prime}(0)=\sum_{n}\left(\varphi_{j_{n+1}}^{\prime}(0)-\varphi_{j_{n}}^{\prime}(0)\right) \sim \sum_{n}\left|Q_{j_{n}}\right|=\sum_{j}\left|Q_{j}\right|$, and (2.2) is proven.

Remark: In the next section we will use this stopping time construction with a q-adic decomposition instead of the dyadic one. It is clear that the estimates of this section, in particular (2.2) and (2.3), remain valid.

\section{Proof of (1.6)}

We will use a standard construction of simply connected domains, based on the following univalence criterion due to Becker [1]:

Theorem 2. If $\varphi$ is analytic in $\mathbb{D}$ and if

$$
\left(1-|z|^{2}\right)\left|z \frac{\varphi^{\prime \prime}(z)}{\varphi^{\prime}(z)}\right| \leq k \leq 1
$$

for all $z \in \mathbb{D}$, then $\varphi$ is injective in $\mathbb{D}$; if moreover $k \in(0,1)$ in (3.1) then $\varphi$ has a quasiconformal extention to the plane. 
We define $\varphi$ by

$$
\varphi(z)=\int_{0}^{z} e^{g(u)} d u, g(z)=\alpha \sum_{n \geq 1} z^{q^{n}}
$$

where $q$ is an integer $\geq 3$. We choose $\alpha, q$ according to the following result of Pommerenke ([4] p.189):

Proposition 3. If

$$
B_{q}=\max _{0 \leq x \leq 1}\left\{2 \sum_{-\infty}^{+\infty} q^{n+x} \exp \left(-q^{n+x}\right)\right\}
$$

then, if $\alpha<1 / B_{q}$ we have (3.1) with some $k<1$.

From now on we fix $\alpha<1 / B_{q}(q \geq 3$ will be chosen later) and consider the function $\varphi$ defined by (3.2). We use the following analogy between lacunary series and sums of independent random variables:

Proposition 4. For every integer $q \geq 2$ there exists a constant $K(q) \geq 0$ such that $\forall t \in[0,2 \pi[, \forall n \geq 0$,

$$
\left|\sum_{k=0}^{n} e^{i q^{k} t}-\sum_{k=0}^{\infty}\left[\left(1-q^{-n}\right) e^{i t}\right]^{q^{k}}\right| \leq K(q) .
$$

The functions playing the role of independent variables are the functions $X_{k}(t)=$ $\cos \left(q^{k} t\right)$.We put $S_{n}=\alpha\left(X_{1}+. . X_{n}\right)$ and define $S_{n}^{\prime}$ as the function that has the constant value $\frac{1}{|T|} \int_{I} S_{n}$ on each dyadic interval $I$ of order $n$. The functions $S_{n}$ and $S_{n}^{\prime}$ are essentially equal in the sense that $\left|S_{n}-S_{n}^{\prime}\right| \leq C$ independently of $n$. For $z=\left(1-q^{-n}\right) e^{i t}$ we thus have $\left(1-|z|^{2}\right)\left|\varphi^{\prime}(z)\right| \sim \exp \left(\alpha S_{n}^{\prime}(t)-n \log q\right)$. Now consider the dyadic decomposition of the disc and let $T(Q)$ be the top $\frac{q-1}{q}$ th of $Q$, so that $Q \backslash T(Q)$ consists of $q$ subcubes. Setting

$$
\epsilon=e^{-N},
$$

we replace the stopping time of the last section by

$$
T_{N}=\inf \left\{n \geq 0 ; S_{n}^{\prime}-n \log q \leq-N\right\} .
$$

By the above arguments and (2.3) we have

$$
\left|\mathbb{D} \backslash D_{\epsilon}\right| \geq c E\left(q^{-T_{N}}\right),
$$

where $E$ stands for expectation, which is the integral over the circle. We define $M_{n}(\theta)=E\left(e^{\theta S_{n}}\right)$. 
Lemma 3.1. For every $\theta \in \mathbb{R}$ there exists $C \geq 1$ such that

$$
\forall n, k \geq 1, \frac{1}{C} M_{n}(\theta) M_{k}(\theta) \leq M_{n+k}(\theta) \leq C M_{n}(\theta) M_{k}(\theta) .
$$

Proof:

$$
E\left(e^{\theta S_{n+k}}\right)=\sum_{I \in \mathcal{I}_{n}} \int_{I} e^{\theta S_{n}} e^{\theta\left(S_{n+k}-S_{n}\right)} d t
$$

where $\mathcal{I}_{n}$ stands for the set of q-adic intervals of order $n$. On each $I \in \mathcal{I}_{n}$ the function $e^{\theta S_{n}}$ is approximately constant so that

$$
E\left(e^{\theta S_{n+k}}\right) \sim \sum_{I \in \mathcal{I}_{n}} \frac{1}{|I|} \int_{I} e^{\theta S_{n}} \int_{I} e^{\theta\left(S_{n+k}-S_{n}\right)} .
$$

In the second integral we change variables and put $u=q^{n} t$ to get

$$
\int_{I} e^{\theta\left(S_{n+k}-S_{n}\right)}=|I| M_{k}(\theta)
$$

and the lemma follows by summation over $\mathcal{I}_{n}$.

Remark: We may assume that $C$ is independent of $\theta$ if $\theta$ belongs to a bounded interval.

Lemma 3.2. For any bounded interval $K \subset \mathbb{R}$ there exists $C>0$ such that for any $\theta \in K$

$$
\int e^{\theta S_{T_{N}}-\log M_{T_{N}}(\theta)} d t \geq C
$$

Proof: Since $\varphi$ is bounded, the variable $T_{N}$ is bounded. Thus there exists $k_{N} \in \mathbb{N}$ (depending on $t$ ) such that $T_{N}+k_{N}=M=$ constant. We may then write, for $I \in \mathcal{I}_{n}$ on which $T_{N}=n$ and for $k=k_{N}$,

$$
\begin{gathered}
\int_{I} e^{\theta S_{n+k}-\log M_{n+k}(\theta)} \sim \frac{1}{|I|} \int_{I} e^{\theta S_{n}} \int_{I} e^{\theta\left(S_{n+k}-S_{n}\right)-\log M_{n+k}(\theta)} \\
\sim \int_{I} e^{\theta S_{n}} \frac{M_{k}(\theta)}{M_{n+k}(\theta)} \sim \int_{I} e^{\theta S_{n}-\log M_{n}(\theta)}
\end{gathered}
$$

by Lemma 3.1, and Lemma 3.2 follows by summation over all q-adic intervals on which $T_{N}$ is constant.

We may now conclude the proof of (1.6). By Lemma 3.2

$$
\int e^{\theta\left(S_{T_{N}}-T_{N} \log q\right)+\theta T_{N} \log q-\log M_{T_{N}}(\theta)} \geq C,
$$

and (3.4) implies

$$
\int e^{\left(\theta \log q-\frac{\log M_{T_{N}}(\theta)}{T_{N}}\right) T_{N}} \geq C e^{\theta N}
$$


Thus (2.1) and (3.5) show that

$$
r(U, 0)-r\left(U_{\epsilon}, 0\right) \geq \epsilon^{-\theta}
$$

provided that $\theta$ satisfies

$$
\theta \log q-\frac{\log M_{T_{N}}(\theta)}{T_{N}} \leq-\log q .
$$

To find such a value $\theta$, we apply the following theorem of Rohde ([4] p.191):

Theorem 3. There exists $C>0$ such that if $q \geq 3, n \geq 0$ and $\theta \in[-1,0]$ then

$$
M_{n}(\theta) \geq C e^{n \log \left(I_{0}(\alpha \theta)\right)}
$$

where $I_{0}(x)=\frac{1}{\pi} \int_{0}^{\pi} \operatorname{ch}(x \cos t) d t$.

It follows that every $\theta \in(-1,0)$ satisfying $\log \left(I_{0}(\alpha \theta)\right)=(\theta+1) \log q$ also satisfies (3.8). The existence of such $\theta$ is immediate from the intermediate value theorem. Moreover, using the computations on p.190 of [4], we find numerically, choosing $q=11, \alpha=1 / B_{q}$, that one can take $\theta=-0.91$.

\section{Proof of (1.5)}

We fix $\varphi \in S$ and write $r_{n}=1-2^{-n}$. We recall that $U=\varphi(\mathbb{D})$ and $U_{\epsilon}=\varphi\left(D_{\epsilon}\right)$. Consider the dyadic decomposition of the disk and denote $\mathcal{W}$ the collection of all $T(Q)^{\prime} s$ that meet $\partial D_{\epsilon}$.

Lemma 4.1. There exists $C \geq 1$ such that

$$
\frac{1}{C} \sum_{\mathcal{W}}|T(Q)| \leq\left|\mathbb{D} \backslash D_{\epsilon}\right| \leq C \sum_{\mathcal{W}}|T(Q)| .
$$

Proof: The first inequality follows from (2.3), and the second inequality is obvious with $C=2$ since every point in $\mathbb{D} \backslash D_{\epsilon}$ is in some $Q$ such that $T(Q) \in \mathcal{W}$.

For $n \geq 1$ let $m_{n}=m_{n}(\epsilon)$ be the number of $T(Q)^{\prime} s$ in $\mathcal{W}$ with order $n$, so that

$$
\left|\mathbb{D} \backslash D_{\epsilon}\right| \sim \sum_{n \geq 1} m_{n} 2^{-2 n} .
$$

For $z \in T(Q) \in \mathcal{W}$ we have $\left|\varphi^{\prime}(z)\right|^{p} \sim \epsilon^{p}(1-|z|)^{-p}$. Writing $r_{n}=1-2^{-n}$, we deduce that

$$
m_{n} \epsilon^{p} 2^{n p} 2^{-n} \leq C \int_{|z|=r_{n}}\left|\varphi^{\prime}\right|^{p} \leq C 2^{n\left(\beta_{\varphi}(p)+o(1)\right)}
$$


where

$$
\beta_{\varphi}(p)=\limsup _{r \rightarrow 1-0} \frac{\log \left(\int_{|z|=r}\left|\varphi^{\prime}\right|^{p}\right)}{\log \left(\frac{1}{1-r}\right)} .
$$

More precisely, for any $\alpha>0$ and for all $n \geq N(\alpha)$, (4.2) is true with $o(1)$ replaced by $\alpha$.

We obtain from (4.2) that $m_{n} \leq C \epsilon^{-p} 2^{(1+\beta-p) n}$ for any $\beta>\beta_{\varphi}(p)$ and $\epsilon$ small enough. Together with (4.1) we obtain

$$
\left|\mathbb{D} \backslash D_{\epsilon}\right| \leq C \epsilon^{-p} \sum_{n \geq 1} 2^{(\beta-p-1) n} \leq C \epsilon^{-p}
$$

if $\beta-p-1<0$. By a result of Clunie and Pommerenke ([4], p.178),

$$
\beta_{\varphi}(p) \leq p-\frac{1}{2}+\left(4 p^{2}-p+\frac{1}{4}\right)^{\frac{1}{2}}
$$

for all $p \in \mathbb{R}$. An easy computation then shows that

$$
\beta_{\varphi}(p)-p-1<0 \quad \text { if } \quad p>\frac{1-\sqrt{33}}{8}=-0.59 . .
$$

This proves (1.5).

We finish by establishing the sharp exponent in Theorem 1 , assuming the validity of Kraetzer's conjecture ([2]) asserting that $\beta(p)=\sup \left\{\beta_{\varphi}(p): \varphi \in\right.$ $S, \varphi$ bounded $\}=p^{2} / 4$ for all $p \in[-2,2]$. Throughout the remainder of the paper, we will thus assume that $\beta(p)=p^{2} / 4$.

First, using $\beta_{\varphi}(p) \leq p^{2} / 4$ instead of the Clunie-Pommerenke estimate in (4.3), we get $\beta_{\varphi}(p)-p-1 \leq \frac{p^{2}}{4}-p-1$ and this last quantity is negative for $-2(\sqrt{2}-1)=$ -0.828 . $<p<0$. It follows that for any simply connected domain $U$ with $r(U, 0)=1$ and for all $\alpha>0, r(U, 0)-r\left(U_{\epsilon}, 0\right) \leq C_{\alpha} \epsilon^{2(\sqrt{2}-1)-\alpha}$.

Conversely, we will show that for any $\alpha>0$ there is a domain $U$ such that $r(U, 0)-r\left(U_{\epsilon}, 0\right) \geq C_{\alpha} \epsilon^{2(\sqrt{2}-1)+\alpha}$. Set $p=-2(\sqrt{2}-1)$. It is known (fractal approximation, see [3]) that for any $\delta>0$ there exists a conformal map $\varphi$ with $\varphi^{\prime}(0)=1$ such that

$$
\int_{|z|=r_{n}}\left|\varphi^{\prime}\right|^{p} \geq C_{\delta} 2^{n(\beta(p)-\delta)} \quad \text { for all } n \geq 1
$$

Using Koebe we then write

$$
\int_{|z|=r_{n}}\left|\varphi^{\prime}\right|^{p} \sim \sum_{k} 2^{-n}\left|\varphi^{\prime}\left(z_{k}\right)\right|^{p}
$$


where the $z_{k}^{\prime} s$ are the dyadic points of order $n$ on $\left\{|z|=r_{n}\right\}$. Fixing a small positive number $\gamma>0$, we partition the set of $z_{k}^{\prime} s$ into the sets $\mathcal{B}, \mathcal{A}_{j}$, where

$$
\mathcal{B}=\left\{z_{k}:\left|\varphi^{\prime}\left(z_{k}\right)\right|^{-1} \leq 1\right\}, \mathcal{A}_{j}=\left\{\left|\varphi^{\prime}\right|^{-1} \in\left[2^{n j \gamma}, 2^{n(j+1) \gamma}\right)\right\}, 0 \leq j \leq \frac{2}{\gamma} .
$$

We may write $\sum_{k} 2^{-n}\left|\varphi^{\prime}\left(z_{k}\right)\right|^{p}=\sum_{k \in \mathcal{B}}+\sum_{j} \sum_{k \in \mathcal{A}_{j}}$. Since $\sum_{\mathcal{B}} \leq 1$ there must exist $j$ such that

$$
\sum_{k \in \mathcal{A}_{j}} 2^{-n}\left|\varphi^{\prime}\left(z_{k}\right)\right|^{p} \geq C \gamma \int_{|z|=r_{n}}\left|\varphi^{\prime}\right|^{p} \geq C \gamma 2^{n(\beta(p)-\delta)} .
$$

We fix such a $j=j_{n}$ and denote by $m_{n}$ the number of elements of $\mathcal{A}_{j}$. Setting $a=a_{n}:=j \gamma$ we then have

$$
m_{n} 2^{-n} 2^{-n(j+1) \gamma p} \geq C \gamma 2^{n(\beta(p)-\delta)} .
$$

Consequently

$$
m_{n} \geq 2^{-n(-\beta(p)-1-a p+2 \delta)}
$$

if $\gamma$ is chosen small enough and $n$ is large. On the other hand, for $k \in \mathrm{A}_{j}$,

$$
2^{-n}\left|\varphi^{\prime}\left(z_{k}\right)\right| \leq 2^{-n} 2^{-n j \epsilon}=2^{-n(1+a)}=: \epsilon_{n} .
$$

Therefore

$$
\left|\mathbb{D} \backslash D_{\epsilon_{n}}\right| \geq C m_{n} 2^{-2 n}=C \epsilon_{n}^{\lambda}
$$

where

$$
\lambda=\lambda_{n}=\frac{1-p a-\beta(p)+2 \delta}{1+a} .
$$

Since $\frac{1-p a-\beta(p)}{1+a} \equiv-p$, the claim follows by choosing $\delta$ arbitrarily small.

\section{References}

[1] J. Becker, Loewnersche Differentialgleichung und quasikonform fortsetzbare schlichte Funktionen, J. Reine Angew. Math. 255 (1972), 23-43.

[2] P. Kraetzer, Experimental bounds for the universal integral means spectrum of conformal maps, Complex Variables Theory Appl. 31 (1996), 305309 .

[3] N. Makarov, Fine structure of harmonic measure, St. Petersburg Math. J. 10 (1999), 217-268

[4] Chr. Pommerenke, Boundary behaviour of conformal maps, Springer, 1991. 